Journal of Sustainable Development of Transport and Logistics

journal home page: https://jsdtl.sciview.net

Mavromatis, S., \& Matragos, V. (2021). Grade impact investigation during stopping sight distance determination on 3D road environment. Journal of Sustainable Development of Transport and Logistics, 6(1), 72-80. doi:10.14254/jsdtl.2021.6-1.6.

\title{
Grade impact investigation during stopping sight distance determination on 3D road environment
}

\section{Stergios Mavromatis $\mathbb{B}^{\mathbb{D}}$, Vassilios Matragos}

Department of Transportation Planning and Engineering, National Technical University of Athens, 5 Heroon Polytechniou Str., GR-15773 Athens stemavro@central.ntua.gr; vasmatragos@mail.ntua.gr

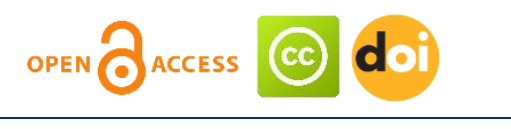

\section{Article history:}

Received: March 08, 2021

1st Revision: March 22, 2021

Accepted: April 23, 2021

\section{DOI:}

10.14254/jsdtl.2021.6-1.6

\begin{abstract}
In the present research, Stopping Sight Distance (SSD) adequacy is assessed through a three-dimensional approach, which associates road visibility in terms of grade effect. SSD adequacy is controlled through the difference between the available and the required SSD. The research is focused on a right turned, two lane rural road, associated with a crest vertical curve for a given speed value. The road design is in accordance with the Greek Road Design Guidelines (OMOE-X, 2001) by utilizing the control design parameters for a design speed of $80 \mathrm{~km} / \mathrm{h}$ and a wide range of grade values, where the vertex point of the crest vertical curve is positioned at fixed points along the road axis. The investigation is based on a vehicle speed exceeding the design speed by $20 \mathrm{~km} / \mathrm{h}$. In total 1874 cases of compound alignments were examined and the results revealed that the available sight distance is decreasing while grade increases. The vehicle speed on the tangent sections was proved unacceptable in terms of providing SSD adequacy. However, it was found that SSD adequacy is granted when the vertical vertex falls inside the circular arc of the curve's horizontal alignment, while the optimum area is defined shortly before the horizontal vertex point.
\end{abstract}

Keywords: stopping sight distance, 3D road environment, grade.

\section{Introduction}

The key objective of a road design is to achieve safety and traffic flow quality. The road geometric parameters should be combined in such a way thus delivering a road environment that satisfies drivers' needs as well as expectations in terms of safety with a fairly constant, low mental workload. The parameter, among others, which constitutes the road alignment predictable and determines a driver's decision, is sight distance, or in other words the road area exposed at any time in the driver's field of vision.

Corresponding author: Vassilios Matragos

E-mail: vasmatragos@mail.ntua.gr

This open access article is distributed under a Creative Commons Attribution (CC-BY) 4.0 license. 
The minimum Sight Distance known as Stopping Sight Distance (SSD) is a highway geometric design control of fundamental importance. As far as safety is concerned, SSD must be provided at every point along the road surface since it directly affects the design values of critical road elements such as vertical curvatures (crest and sag) as well as the middle ordinate values, due to roadside obstacles, on left curved divided highways and right curved sections of two lane rural roads.

The objective of the present research is to determine SSD adequacy in a three-dimensional road environment, where the roadway's grade impact is investigated. The suggested approach ensures far more accurate results, compared to current practice where SSD adequacy is assessed through a separate and independent evaluation process in horizontal and vertical alignment respectively. More specifically, for a given horizontal road geometry, the authors, in terms of SSD adequacy, intend to assess the effect of the crest vertical curve's vertex arrangement.

\section{SSD determination on 3D road environment}

In highway engineering, under the similar lighting conditions, the following two SSD values are reported:

- SSD $_{\text {REQUIRED }}$ related with the ability of the vehicle to reach stop condition and depends on

$\circ$ the road, in terms of geometry

$\circ$ the driver, in terms of perception - reaction utilization

- the vehicle, in terms of provided dynamic characteristics

- $\mathrm{SSD}_{\mathrm{AVAILABLE}}$ associated with the frontal sight field visible to the driver during daytime conditions and depends on

$\circ$ the roadside environment (roadside obstacles)

$\circ$ road geometry

SSD adequacy is granted when:

$\mathrm{SSD}_{\text {REQUIRED }} \leq \mathrm{SSD}_{\text {AVAILABLE }}$

According to existing design policies the required SSD consists of two distance components: the distance traveled during driver's perception - reaction time to the instant the brakes are applied (usually the time value is between $2.0 \mathrm{sec}-2.5 \mathrm{sec}$ ) and the distance while braking to stop the vehicle.

However the above approach ignores curved areas of both horizontal and vertical alignment, since, on one hand, the portion of friction provided in the longitudinal direction, assigned to serve the braking process, is associated directly to the friction demanded laterally (Krempel, 1965), and on the other, the grade values involved in vertical curves are variable. In the present paper, the required SSD determination was based on a previous research (Mavromatis et al., 2012), where the above parameters were taken under consideration.

The SSD adequacy analysis is based on either 2-D or 3-D models. The 2-D SSD investigation is rather fragmentary and may produce design deficiencies due to inaccurate calculation of the available sight distance, where even more critical situations might occur. This concern has been identified by many researchers in the past and a wide range of 2-D and 3-D approaches have been developed to address the problem. One of the first researchers (Sanchez, 1994) that assessed the available sight distance on 3-D alignment studied the interaction between the sight distance and the 3-D combined alignment idealized into a net of triangles.

Several years later, another researcher (Hassan et al., 1996), presented an analytical model for computing available sight distance on combined horizontal and vertical highway alignments, using parametric finite elements (4,6 and 8-node rectangular elements as well as 3-node triangular elements) to represent the highway and sight obstructions. The idea behind the proposed model is summarized in checking the driver's sight line, which is represented by a straight line between the driver's eye and an object, against all the possible sight obstructions, by using an iterative procedure.

In the past years, in order to evaluate the actual sight distance in real driving conditions, a number of 3-D models are found in the literature (Moreno, 2010; García, 2004; Ismail \& Sayed, 2007; Romero \& García, 2007; Yan et al., 2008; Zimmermann, 2005; DiVito \& Cantisani, 2010). which base their performance through the correlation between the road surface, the ground terrain and the roadside environment aiming to optimize the available sight distance. Therefore, either new algorithms or design 
parameter combinations are introduced, ignoring in many cases the topographic visual restraints. Moreover, none of the above-mentioned approaches suggested a comprehensive methodology to simulate from a 3-D perspective concurrently both the cross-section design and the vehicle dynamics in space during emergency braking conditions.

The objective of this work is to investigate SSD adequacy while utilizing control geometric parameters based on Greek OMOE-X Design Guidelines (OMOE-X, 2001). The applied methodology is based on identifying areas of interrupted vision lines between driver - obstacle being less than the required distance necessary to bring the vehicle to a halt.

The available as well as required SSD values are defined through the difference of the road stations between starting and ending points, assumed at any desired axis offset and equal to the distance between the road's centerline and, usually, half of the examined lane width. The above process, illustrated on the flow chart of Figure 1, is incorporated in H12 (NTUA, 2012) road design software.

Figure 1: Flowchart of SSD adequacy investigation

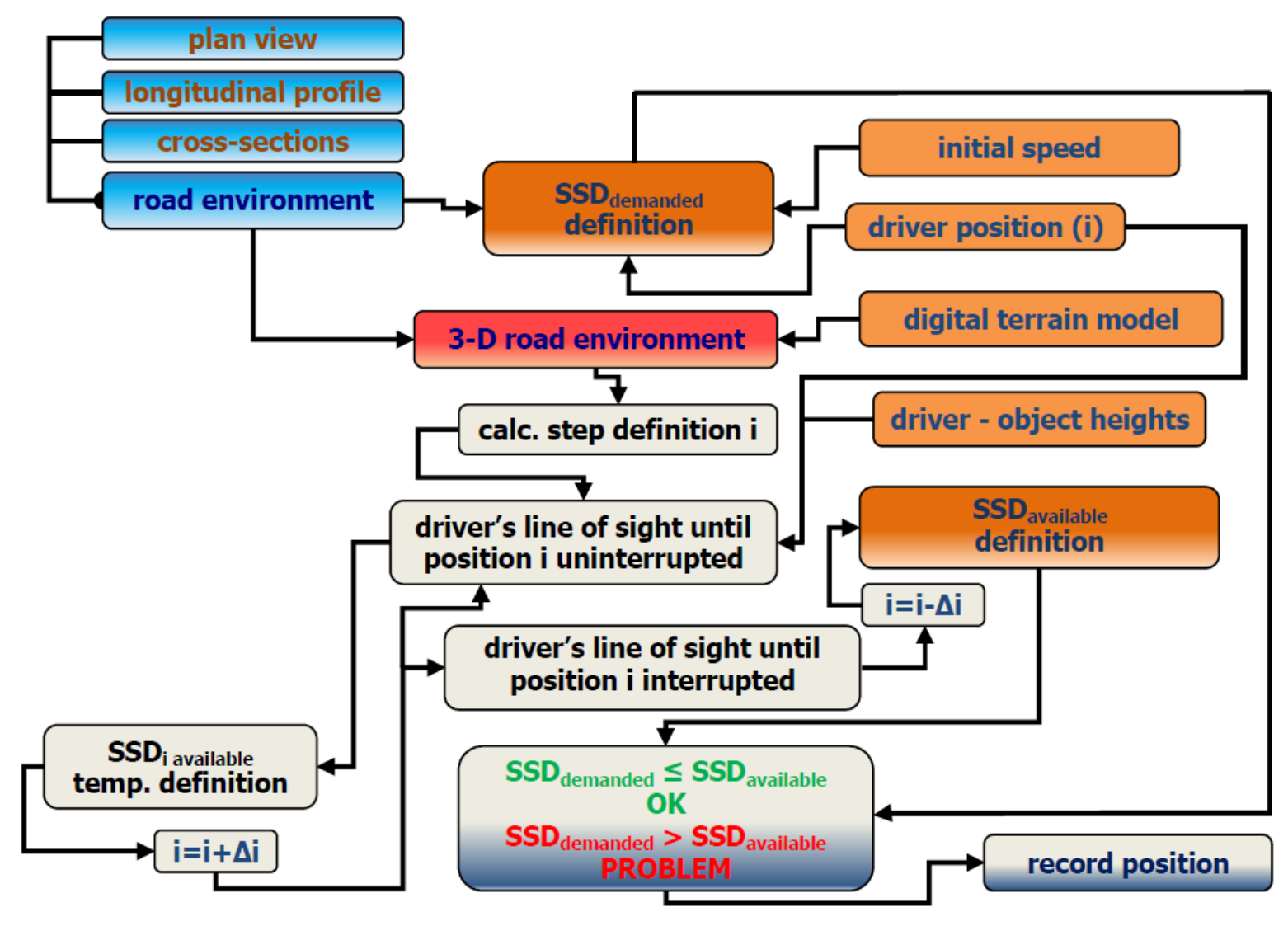

\section{SSD adequacy investigation on 3D road environment}

\subsection{Road and vehicle parameter selection and outputs}

The investigation was performed on a right curved, two lane rural road, for the same horizontal and vertical geometry where the investigation was limited on the road surface environment.

The selection of the particular road class was due to the extremely critical situations that may rise in cases of compound alignments, where the preceding horizontal curve is hidden due to road's vertical profile (Moreno, 2010; FGSV, 2008). Such case represents an unsuccessful geometric design in 3D road environment, although the two-dimensional approach delivers SSD adequacy.

Although a single horizontal alignment is associated with a single vertical alignment, a number of alignment combinations accrue by positioning ('sliding') the vertex of the crest vertical curve at fixed distances along the horizontal alignment. Moreover, by utilizing the same vertical curvature rate with various preceding and succeeding grade values a large number of combinations accrue for each station of the vertical vertex on the horizontal alignment. 
The SSD adequacy investigation is carried out for a design speed of $80 \mathrm{~km} / \mathrm{h}$ and the relevant control geometric parameters based on OMOE-X Design Guidelines; namely:

- $\mathrm{R}=280 \mathrm{~m}$ (horizontal radius)

- $\mathrm{L}=40 \mathrm{~m}$ (length of horizontal transition curve)

- $\mathrm{H}_{\mathrm{k}}=4500 \mathrm{~m}$ (crest vertical curvature rate)

- Srange $\pm 1 \%$ up to $\pm 7 \%$, (grade range)

The typical cross section was selected b2 type (OMOE-X, 2001), [lane + outer shoulder width = $3.75 m+0.25 m$ (per direction of travel)]. On the other hand, the cross slope was set to $7.0 \%$ and $2.5 \%$ on the circular arc and tangents areas respectively.

Furthermore, the investigation was based on vehicle speed $80+20 \mathrm{~km} / \mathrm{h}$ since such an excess is an acceptable practice, at least from the OMOE-X design guidelines point of view. Finally, the deceleration rate corresponding to the vehicle's speed was $d=3.4 \mathrm{~m} / \mathrm{sec} 2$, the driver's perception - reaction time $2.0 \mathrm{sec}$, and the driver and object heights were set to $1.06 \mathrm{~m}$ and $0.50 \mathrm{~m}$ respectively.

All possible grade combinations were utilized between $\pm 1 \%$ up to $\pm 7 \%$ on a $1 \%$ step basis, where in order to classify the outputs more efficiently, the following cases were utilized:

- Case I, the preceding and succeeding grade values of the vertical curve were the same in terms of absolute values

- Case II, the preceding and succeeding grade values of the vertical curve differed in terms of absolute values

The vertical vertex was positioned along the horizontal alignment by $50 \mathrm{~m}$ increment step regardless the examined case. Based on the flowchart of Figure 1, the length of the total SSD breakdown zone formed by the difference between SSD $_{\text {REQUIRED }}-$ SSD $_{\text {AVAILABLE }}$ was delivered in 3D road environment.

Figure 2 shows an example regarding SSD adequacy investigation on the above described 3D compound road surface referring to $80+20 \mathrm{~km} / \mathrm{h}$ speed where for the same horizontal alignment, the vertical vertex formed by $s 1=6.00 \%, s 2=-6.00 \%$, is positioned at St.700. The horizontal axis represents the road stations where the horizontal and vertical curvatures are projected linearly. The vertical axis illustrates two different SSD values corresponding to the stations of the compound alignment shown horizontally; namely SSD REQUIRED $_{\text {(continuous line) and SSD }}$ AVAILABLE (dashed line). A closer look of Figure 2 reveals an under-design zone, located at the area where the continuous line ( $\mathrm{SSD}_{\text {REQUIRED }}$ ) overlaps the dashed one ( $S_{S D}$ AVAILABLE). In terms of quantifying the SSD safety violation area between the SSD REQUIRED and the SSD $_{\text {AVAILABLE, }}$ the total SSD breakdown zone, or in other words the length of hidden roadway to the driver, is approximately $175 \mathrm{~m}$.

Figure 2: SSD adequacy investigation for a single compound alignment

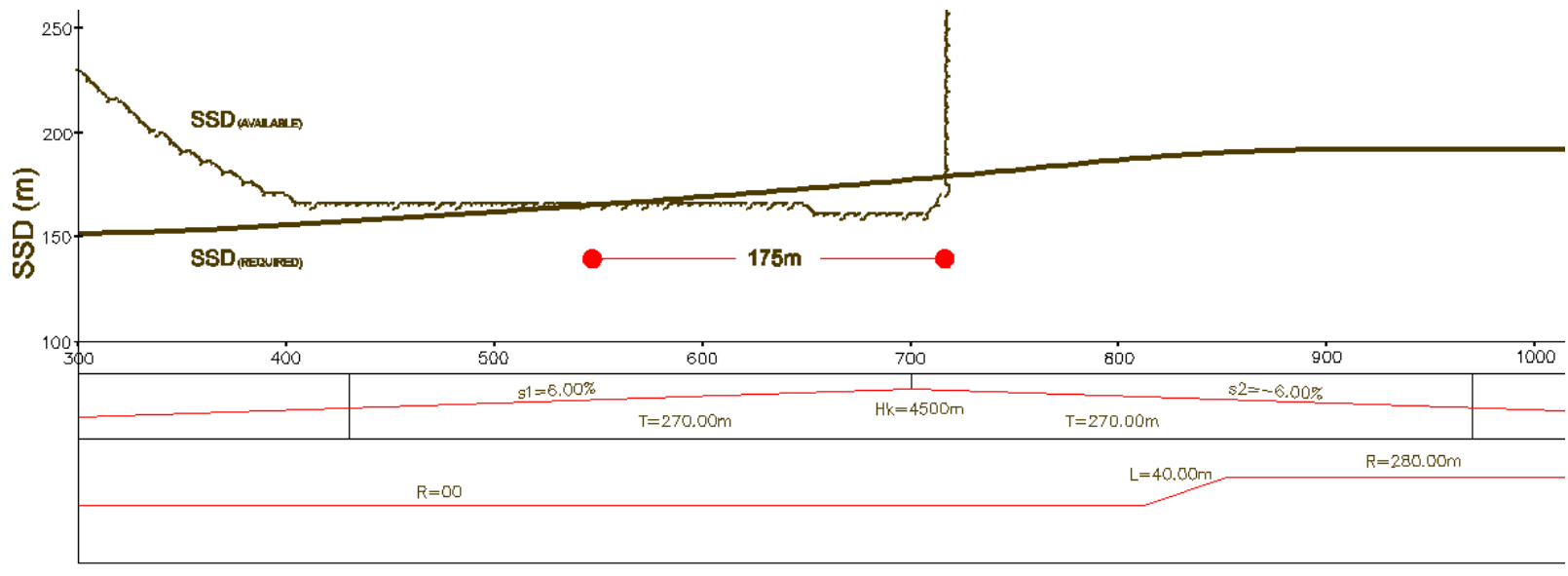

Note: Vertical Vertex St.700, Start of Vertical Curve St.430 (700-270), End of Vertical Curve St.970

$(700+270)$.

Figure 3 quantifies the SSD inadequacy for the entire grade values utilized in Case I, while Figure 4 shows the relevant findings for Case II. In both figures, the horizontal alignment is projected linearly where, as already stated, the various lines are formed by "sliding" vertical vertex by $50 \mathrm{~m}$ increment step. For example, in Figure 3 (Case I), it can be seen that for $s= \pm 6 \%$ at station 700 of the horizontal alignment, the difference between $S_{S D}$ REQUIRED $-S_{\text {AVAILABLE, }}$ is $175 \mathrm{~m}$ approximately, as shown in Figure 2. 
Therefore, Figure 3 illustrates 269 different alignment arrangements (Case I), while 1605 alignment combinations were evaluated for Case II and are shown in Figure 4. However, due to the density of lines assessed through Case II, Figure 4 illustrates the grade combinations for max grade values up to $\pm 5 \%$. In both figures the areas of the horizontal transition curves are shaded in grey. In other words, between the grey columns lies the circular arc projected linearly. It is obvious that areas with SSD adequacy correspond to zero length of hidden roadway.

Figure 3: SSD adequacy investigation (Case I)



Figure 4: SSD adequacy investigation (Case II)

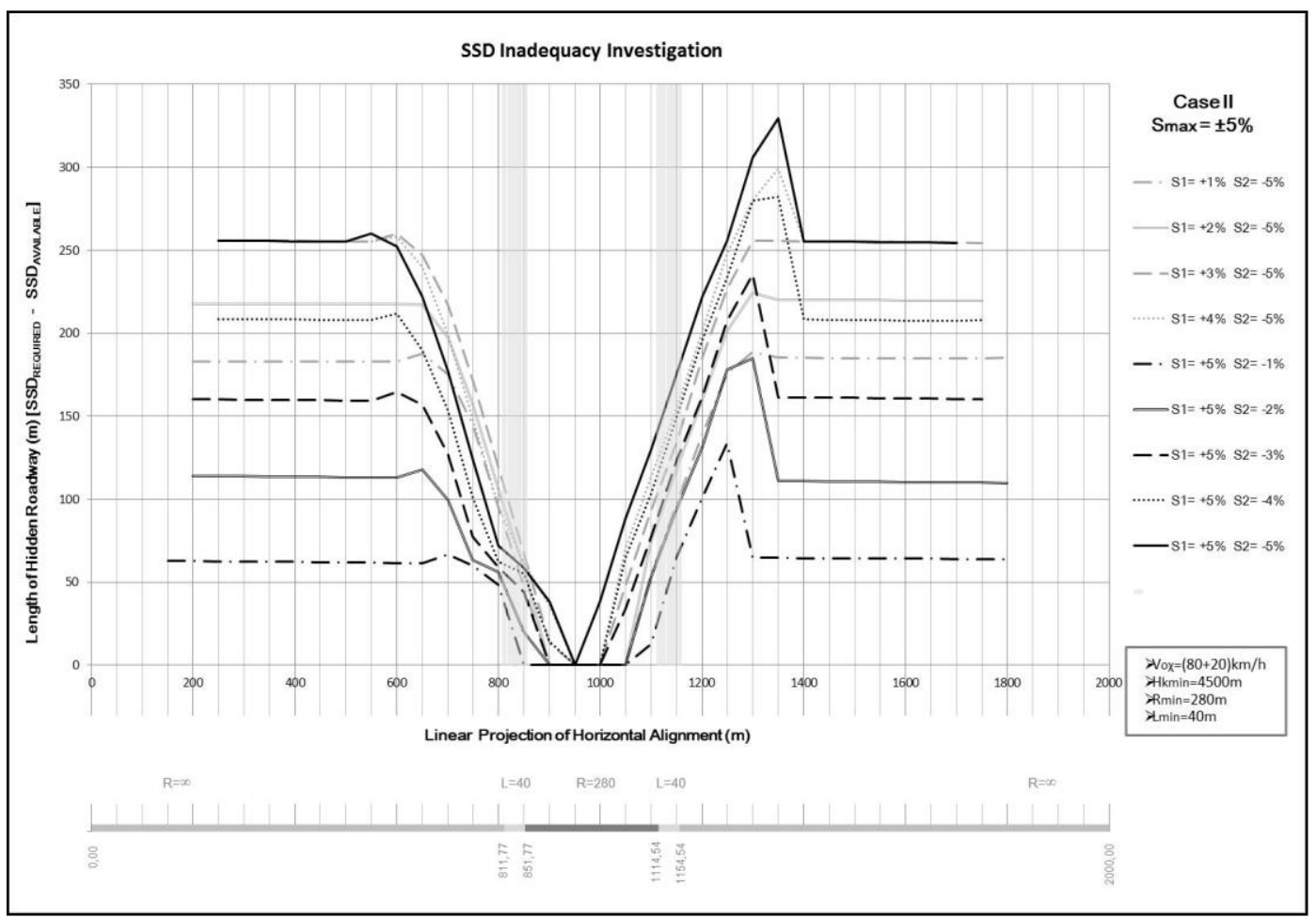




\subsection{Results analysis}

In both Figure 3 and Figure 4 it can be seen that as the vertical vertex approaches the horizontal curve area, the SSD inadequacy length, formed by the difference between SSD REQUIRED $_{-}$SSD $_{\text {AVAILABLE }}$ decreases, thus reducing the SSD breakdown zone. This finding confirms relevant suggestions of the Green Book (AASHTO, 2011) where it is stressed that in order SSD provision to be granted, the vertical curve should be entirely designed inside the horizontal curve. More or less, similar suggestions are provided through the Spanish Design Guidelines (Ministerio de Fomento, 2000) as well, where the desired horizontal - vertical curve arrangement is reached when the vertical crest curve falls completely inside the horizontal curve including spirals.

Another crucial finding, in both figures, is the fact that when the SSD adequacy evaluation is examined at the tangent area of the horizontal alignment, without the impact of the vertical curvature, the safety violation in terms of SSD inadequacy reaches its peak values. This means that the excess of the design speed by $20 \mathrm{~km} / \mathrm{h}$ delivers critical safety considerations on tangents, and as a result, a lower posted speed is required at least when designing based on the control values associated to the speed of $80 \mathrm{~km} / \mathrm{h}$.

However, certain areas with abrupt value alterations just after the horizontal curve, refer to the existence of more than one overlapping zones between $S_{S D_{\text {REQUIRED }}}$ and SSD $_{\text {AVAILABLE }}$ values (Mavromatis et al., 2012).

The SSD breakdown zone areas, once again in both figures, seem rather symmetrical to a vertical axis just before the horizontal vertex (mid-point of the horizontal arc). This finding confirms a relevant research study (Moreno, 2010), according to which the location of the vertical midpoint that maximizes available sight distance is located before the horizontal vertex.

\subsection{SSD adequacy}

SSD provision is an essential requirement during road design. Therefore, the assessed alignment arrangements with SSD adequacy for both Case I and Case II are shown through Figure 5. The alignment, in order to be more easily overviewed, is once again projected linearly. In Figure 5, the alignments referring to Case II are further separated to the ones where the absolute value of the maximum grade refers to upgrade (Case II A) and downgrade (Case II B) conditions respectively

The grade values of $\pm 1 \%$ referring to Case I are not shown, since all the examined alignments with such vertical geometry were found safe in terms of SSD provision.

Figure 5 once again confirms the empirical suggestions of the Green Book (AASHTO, 2011), as well as the relevant Spanish Design Guidelines (Ministerio de Fomento, 2000), according to which SSD adequacy is granted when the vertical curvature falls inside the horizontal curve. 
Figure 5: SSD adequacy areas
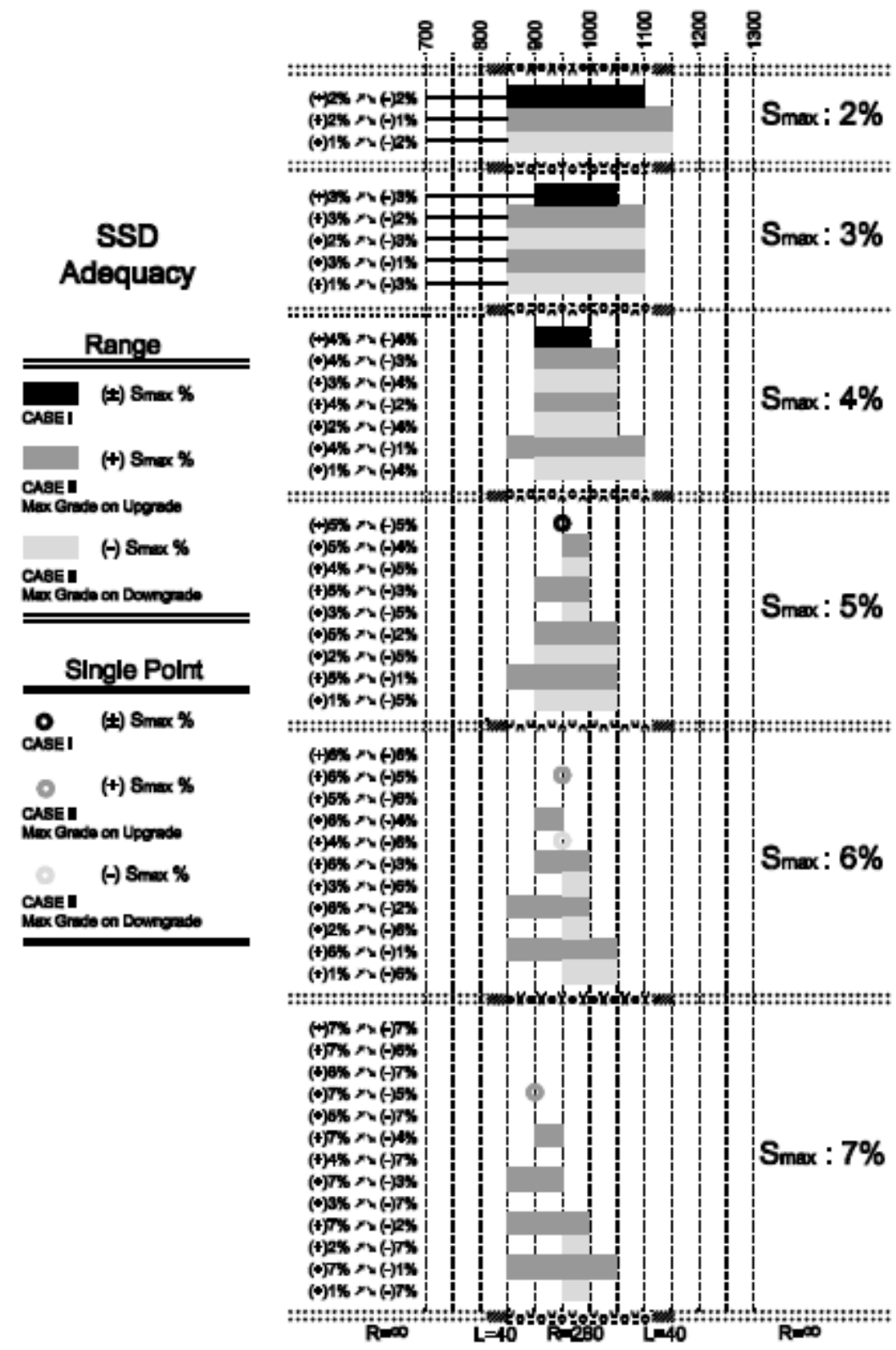

\section{Conclusions}

The present paper investigates, in terms of SSD provision, the impact of crest vertical geometry, against a right curved road section of a two-lane rural road. The road geometry was based on control values referring to a design speed of $80 \mathrm{~km} / \mathrm{h}$, where the vehicle speed exceeded the design speed by $20 \mathrm{~km} / \mathrm{h}$.

Although a single alignment in terms of absolute geometry values was utilized, the positioning of the vertical vertex at fixed distances along the horizontal alignment, and moreover, the various preceding and succeeding grade values assessed for the same vertical curvature rate, delivered a large number of alignment combinations.

As far as the tangent sections are concerned, the vehicle speed on the tangent sections was proved unacceptable in terms of providing SSD adequacy. The results regarding areas with SSD provision revealed compatible findings compared to empirical considerations, according to which the road's vertical curve should be contained within the curve of the horizontal alignment.

Furthermore, it was found that SSD adequacy is granted when the vertical vertex falls inside the circular arc of the curve's horizontal alignment, while the optimum area is defined shortly before the horizontal vertex point. 
The present work is a trigger for further investigation and is indicative of the size and depth of analysis required to assess SSD adequacy investigation in three-dimensional road environment.

\section{Citation information}

Mavromatis, S., \& Matragos, V. (2021). Grade impact investigation during stopping sight distance determination on 3D road environment. Journal of Sustainable Development of Transport and Logistics, 6(1), 72-80. doi:10.14254/jsdtl.2021.6-1.6

\section{References}

American Association of State Highway and Transportation Officials (AASHTO). (2011). A Policy on Geometric Design of Highways and Streets 6th Edition. Washington, DC.

DiVito, M., \& Cantisani, G. (2010). D.I.T.S.: A software for sight distance verification and optical defectiveness recognition. In 4th International Symposium on Highway Geometric Design TRB, Valencia Spain.

FGSV-Forschungsgesellschaft für Straßen- und Verkehrswesen. (2008). Hinweise zur Visualisierung von Entwürfen für außerörtliche Straßen. FGSV, Köln: H ViSt.

García, A. (2004). Optimal vertical alignment analysis for highway design - discussion. Journal of Transportation Engineering, 130(1), 138.

Hassan, Y., Easa, S. M., \& Abd El Halim, A. O. (1996). Analytical model for sight distance analysis on threedimensional highway alignments. Transportation Research Record, 1523(1), 1-10.

Ismail, K., \& Sayed, T. (2007). New algorithm for calculating 3D available sight distance. Journal of Transportation Engineering, 133(10), 572-581.

Krempel, G. (1965). Experimenteller Beitrag zu Untersuchungen an Kraftfahrzeugreifen. Dissertation. TU Karlsruhe.

Mavromatis, S., Palaskas, S., \& Psarianos, B. (2012). Continuous three-dimensional Stopping Sight Distance control on crest vertical curves. Advances in Transportation Studies, (29).17-30.

Ministerio de Fomento, (2000). “Trazado” Instrucción de Carreteras Norma 3.1 - IC. Madrid, Spain.

Ministry of Environment, Regional Planning and Public Works. (2001). Guidelines for the Design of Road Projects (OMOE- $X$ ). Alignment, Part 3, Greece.

Moreno Chou, A. T., Pérez, V. M. F., García, A. G., \& Rojas, M. A. R. (2010). Optimal 3D coordination to maximize the available stopping sight distance in two-lane roads. In 4th International Symposium on Highway Geometric Design Polytechnic University of Valencia Transportation Research Board.

NTUA - National Technical University of Athens. (2012). H12 - Road Design Software, NTUA, Athens, Greece.

Romero, M. A., \& García, A. (2007, January). Optimal overlapping of horizontal and vertical curves maximizing sight distance by genetic algorithms. In The 86th Annual Meeting of the Transportation Research Board. Washington, DC.

Sanchez, E. (1994). Three-dimensional analysis of sight distance on interchange connectors. Transportation Research Record, 1445, 101-108.

Yan, X., Radwan, E., Zhang, F., \& Parker, J. C. (2008). Evaluation of dynamic passing sight distance problem using a finite-element model. Journal of Transportation Engineering, 134(6), 225-235.

Zimmermann, M., \& Roos, R. (2005). Increased safety resulting from quantitative evaluation of sight distances and visibility conditions of two-lane rural roads. In 3rd International Symposium on 
Highway Geometric Design Transportation Research Board American Association of State Highway and Transportation Officials (AASHTO) Federal Highway Administration American Society of Civil Engineers Association Mondiale de la Route International Road Federation Institute of Transportation Engineers (ITE) National Association of County Engineers Transportation Association of Canada (TAC) Chicago Department of Transportation Illinois Department of Transportation Illinois State Toll Highway Authority.

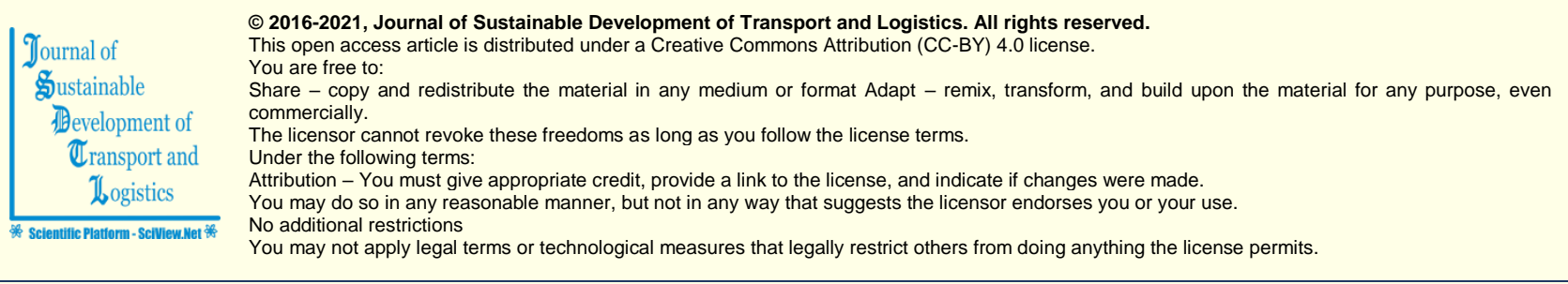

Journal of Sustainable Development of Transport and Logistics (ISSN: 2520-2979) is published by Scientific Publishing House "CSR", Poland, EU and Scientific Publishing House "SciView", Poland, EU

Publishing with JSDTL ensures:

- Immediate, universal access to your article on publication

- High visibility and discoverability via the JSDTL website

- Rapid publication

- Guaranteed legacy preservation of your article

- Discounts and waivers for authors in developing regions

Submit your manuscript to a JSDTL at https://jsdtl.sciview.net/ or submit.jsdt|@sciview.net 